\title{
Neue Therapiestrategien bei kutanen T-Zell-Lymphomen ${ }^{1}$
}

\section{New Treatment Strategies for Cutaneous T-Cell Lymphomas}

Autoren

Institute

\author{
C. Schulz ${ }^{1}$, C. Löser ${ }^{1}$, C. Assaf ${ }^{2}$, E. Dippel
}

1 Hautklinik, Klinikum der Stadt Ludwigshafen

2 Klinik für Dermatologie, Helios Klinikum Krefeld

\section{Bibliografie}

Dol $10.1055 / \mathrm{s}-0028-1119728$

Akt Dermatol 2009; 35:

140-144 @ Georg Thieme

Verlag KG Stuttgart · New York ISSN 0340-2541

Korrespondenzadresse

Prof. Dr. Edgar Dippel

Hautklinik

Klinikum der Stadt

Ludwigshafen $\mathrm{GmbH}$

Bremserstraße 79

67063 Ludwigshafen

dippele@klilu.de

\section{Zusammenfassung \\ $\nabla$}

Kutane T-Zell-Lymphome sind eine Gruppe extranodaler Non-Hodgkin-Lymphome mit großer klinischer und histopathologischer Heterogenität, die sich am Hautorgan manifestieren. Sie zeigen eine große Bandbreite klinischer und histologischer Erscheinungsformen. Aktuelle Ergebnisse aus der Grundlagenforschung bestätigten die Heterogenität und erlaubten tiefere Einblicke in die Tumorbiologie, die Tumorimmunologie und die molekulare Genetik der Lymphome. Dabei ergab sich eine Fülle von neuen Zielstrukturen auf den

\section{Einleitung}

Die neue WHO-EORTC-Klassifikation und die neuen Stadieneinteilungen für kutane T-ZellLymphome haben die Systematik der kutanen Lymphome besser strukturiert und die Übersichtlichkeit einer sehr heterogenen Gruppe von extranodalen Lymphomen verbessert [1 - 3]. Neben der Klassifikation und Stadieneinteilung gibt es auch eine große Zahl von neuen therapeutischen Ansätzen [4]. Dies ist nicht nur durch die molekularbiologischen Erkenntnisse zu den kutanen T-Zell-Lymphomen bedingt, sondern auch durch die allgemeinen Weiterentwicklungen einer zielgerichteten (targeted) Therapie oder sogar einer individualisierten Therapie in der Onkologie beeinflusst [5]. Eine zunehmende Anzahl von potenziellen therapiefähigen Zielstrukturen (Targets) sind insbesondere bei Lymphomen entdeckt worden.

Hilfreich waren hier Genprofil-Analysen oder das Immunoscreening von Plasmamembranproteinen, welche z.B. Unterschiede zwischen den

\footnotetext{
${ }^{1}$ Dieses Manuskript ist dem 10-jährigen Jubiläum der Berliner Stiftung für Dermatologie gewidmet.
}

malignen Lymphomzellen, die es nun ermöglichen, neue Therapiestrategien zu entwickeln. Je genauer die Zuordnung zu den einzelnen Lymphomentitäten und die Erhebung diagnostischer Informationen $\mathrm{zu}$ individuellen Patienten mit kutanen T-Zell-Lymphomen gelingt, umso zielgerichteter kann die Therapie erfolgen. Eine kurative Therapie ist jedoch noch nicht in Sicht, daher bleibt die bisherige allgemeine Behandlungsstrategie der kutanen T-Zell-Lymphome mit einer stadiengerechten Ausrichtung weiterhin erhalten.

T-Zell-Lymphomentitäten Mykosis fungoides und Sézary-Syndrom herausarbeiten [6,7].

Die molekulare Signatur eines T-Zell-Lymphoms wird zur Arbeitsgrundlage, um neue Signaltransduktionswege zu untersuchen und zielgerichtete Interventionen durchzuführen. Bereits weitgehend etabliert in der stadiengerechten Therapie sind das RXR-selektive Retinoid Bexaroten als Monotherapeutikum $[8,9]$ und das Fusionstoxin Denileukin Diftitox, welches über den CD25-Rezeptor mit Freisetzung des Diphteritoxins die Zerstörung der Tumorzelle bewirken soll [10-12]. Noch in der klinischen Prüfung befindlich sind der anti-CD52 Antikörper Alemtuzumab und der anti-CD4 Antikörper Zanolimumab [13]. Eine weitere aussichtsreiche Substanz, welche 2009 in die klinische Prüfung (Phase III) geht, ist der PurinNukleosid-Phosphorylase-Inhibitor Forodesine. Neue Kapitel in der Therapiegeschichte der Lymphome werden durch Substanzen aufgeschlagen, die den Ubiquitin- Proteosomenmetabolismus inhibieren, wie z. B. Bortezomib, und die Histonacetylierung beeinflussen, wie z.B. Vorinostat [14-15]. Ausschlaggebend für die weitere klinische Verwendung dieser Substanzen sind allerdings qualitativ hochwertige klinische Studien. Dies ist eine Herausforderung, da die extranoda- 
len kutanen Lymphome mit vergleichsweise niedriger Inzidenz und hoher Heterogentität in Erscheinung treten. Ein systematischer Ansatz ist hier die zukünftige Therapieplattform der EORTC Cutaneous T-Cell-Lymphoma Task Force (CLTF), die in dieser Übersichtsarbeit auch dargestellt werden soll.

\section{Proteinaseinhibitoren (Bortezomib)}

Ein wesentlicher intrazellulärer Abbauweg von Proteinen ist der Ubiquitin-Proteasomen-Prozessweg. Für die Entdeckung des ATP-abhängigen Degradationsprozesses wurde Ciechanover, Hershko und Rose 2004 der Nobelpreis für Chemie verliehen. Ubiquitin wird in Gegenwart von ATP an ein Proteinsubstrat gebunden und markiert dieses für einen späteren proteolytischen Abbauprozess durch einen multikatalytischen Proteinasekomplex, das 26S Proteasom. Die Hauptkomponente ist das 20S Proteasom, welches für $80-90 \%$ der intrazellulären Proteindegradation verantwortlich ist [16]. Bortezomib ist der erste medikamentöse Hemmer des 26S Proteasoms, der von der FDA zugelassen wurde. Die Wirkung von Bortezomib basiert auf einer Blockade der Stoffwechselwege der malignen Zelle, die sich durch unkontrolliertes Wachstum auszeichnen [17]. Kommt es zu einer Proteasom-Blockade, werden vitale Proteolyse-Prozesse unterdrückt. Nach einmaliger intravenöser Gabe nehmen die Plasmakonzentrationen von Bortezomib biphasisch ab. Die Abnahme ist durch eine schnelle Verteilungsphase, gefolgt von einer langsameren terminalen Eliminationsphase charakterisiert. Die Halbwertzeit beträgt geschätzte 5 bis 15 Stunden. Das Boratom des Bortezomib bindet mit einer hohen Affinität und Selektivität an die katalytische Funktion des 26S Proteasoms.

Auf den ersten Blick erscheint die Inhibition der Proteindegradation ein unspezifischer Prozess, der nicht sofort daran denken lässt, diese Substanz in der Tumortherapie einzusetzen. Doch durch die Entdeckung dieser neuen Substanzgruppe konnten gerade beim Myelom eine große Anzahl von Effekten entdeckt werden, wie die Inhibition von NF-kB, welche nicht nur verantwortlich für Zellproliferation, sondern auch für Chemotherapieresistenz und Apoptoseresistenz sind. Weitere Effekte sind die verminderte Degradation von Zellzyklus-abhängigen Kinaseinhibitoren wie p27 und p21, die Akkumulation von proapoptotischen Faktoren und eine breite Wirkung auf Zytokine und Adhäsionsmoleküle ( $\bullet$ Abb. 1).

Die ersten klinischen Erfahrungen mit Bortezomib bei rezidivierenden oder therapierefraktären kutanen T-Zell-Lymphomen zeigen bereits das therapeutische Potential [18]. Zinani u. Mitarb. behandelten 12 Patienten mit kutanen T-Zell-Lymphomen mit einer Dosis von $1,3 \mathrm{mg} / \mathrm{m}^{2} 2 \times$ wöchentlich für 2 Wochen. Dieser Behandlungszyklus wurde $6 \times$ in 3-wöchigen Abständen durchgeführt. Die Ansprechrate lag bei 67\% (2 komplette, 6 partielle Remissionen). Im Nebenwirkungsprofil waren Grad 3-Toxizitäten beobachtet worden mit Neutropenie $(n=2)$, Thrombozytopenie $(n=2)$ und sensorischer Neuropathie $(n=2)$. Gerade die Neuropathie mit Sensibilitätstörungen an den Händen und Füßen ist therapeutisch schwer zu beeinflussen, bildet sich jedoch nach der Behandlung in der Regel zurück. Weitere größere Studien müssen folgen, um diese Daten zu untermauern.

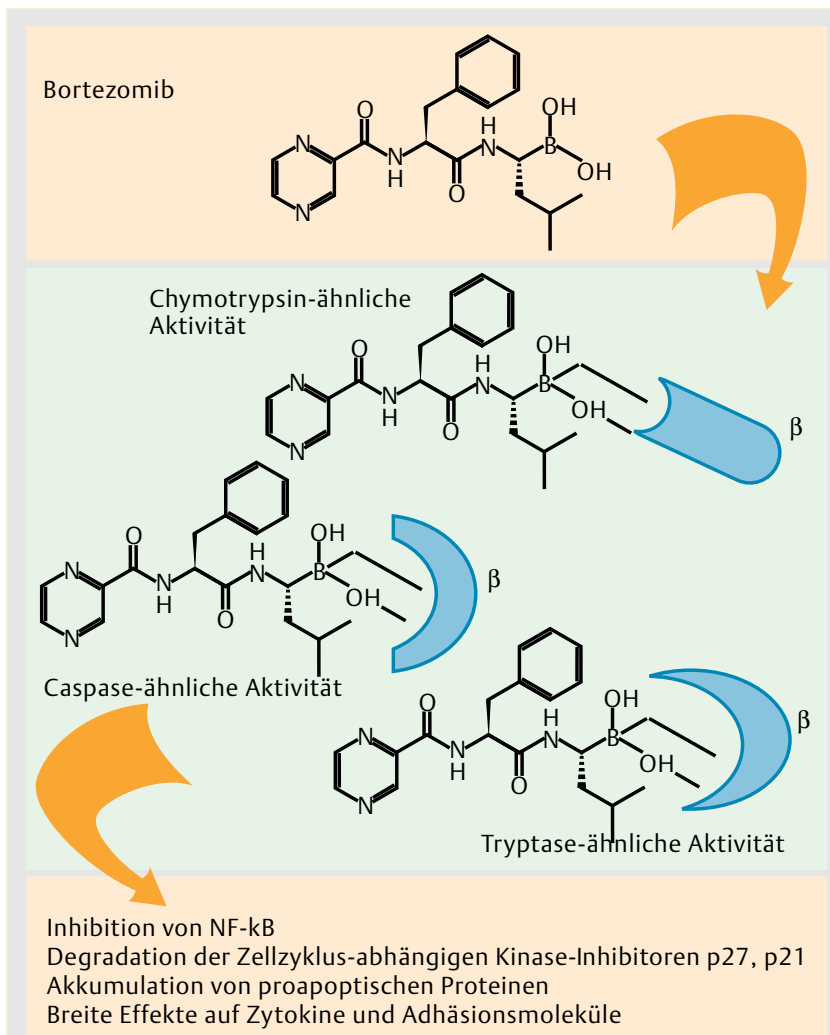

Abb. 1 Effekte von Bortezomib durch Inhibition der unterschiedlichen enzymatischen Aktivitäten des 205 Proteasoms.

\section{Histondeacetylase-Inhibitoren}

$\nabla$

Histondeacetylase-Inhibitoren stellen eine neue Substanzgruppe in der Therapie verschiedener onkologischer Erkrankungen dar.

Die Onkogenese von malignen Zellen wird generell von genetischen und epigenetischen Prozessen beeinflusst. Die genetischen Veränderungen der DNA werden durch Mutationen und Translokationen verursacht, die aufgrund einer veränderten mRNA bzw. eines veränderten Proteins die Zellen transformieren. Der epigenetische Prozess bestimmt, ob eine offene oder geschlossene Chromatinstruktur vorliegt. Der Chromatinzustand wiederum, der über die Acetylierung von Histonen reguliert wird, ist maßgebend dafür, welche Proteine konstitutiv produziert werden. Dieser Mechanismus stellt den Angriffspunkt für die Verwendung von Deacetylierungsinhibitoren dar ( $\bullet$ Abb. 2). In neoplastischen Zellen liegt unter anderem eine Überexpression des Enzyms Histon-Deacetylase vor. Obwohl Histondeacetylaseinhibitoren nach bisherigen Kenntnissen nur einen Teil der Gene beeinflussen [19], sind hierdurch u.a. Tumorsuppressorgene und solche Gene betroffen, die an der Kontrolle von Zellproliferation und -differenzierung sowie Apoptose beteiligt sind [20,21]. Diese Effekte, die durch Histondeacetylaseinhibitoren indirekt vermittelt werden, stellen die Rationale für den Einsatz dieser Substanzen in der onkologischen Therapie dar.

Derzeit sind fünf Gruppen von Histondeacetylaseinhibitoren bekannt ( $\odot$ Tab. 1).

Bisher gibt es Erfahrungen mit Depsipeptid und Vorinostat in der Behandlung kutaner T-Zell-Lymphome. Vorinostat (SAHA) ist der Gruppe der Hydroxamsäuren zuzuordnen und wurde nach Durchführung einer multizentrischen Phase-II-Studie als 


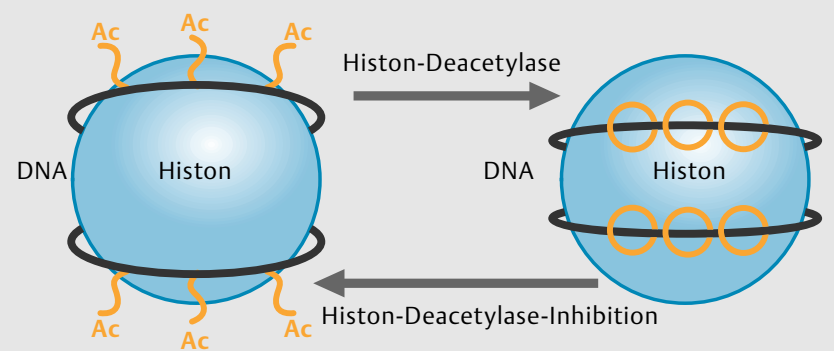

Acetylierte Histone

Deacetylierte Histone

Aktivierung der Gentranskription

Inhibierung der Gentranskription

Zelldifferenzierung, Zyklusarrest, Apoptose, aktivierte

Tumorsuppressorgene

Zellproliferation

Tumorwachstum

Inhibition von Tumorwachstum

Abb. 2 Einfluss des DNA-Acetylierungsstatus auf Gentranskription und Tumorwachstum, $\mathrm{Ac}=$ Acetylierung.

Tab. 1 Klassen der Histondeacetylaseinhibitoren. Substanzen mit Effektivität bei kutanen T-Zell-Lymphomen sind hervorgehoben.

\begin{tabular}{|l|l|}
\hline Hydroxamsäuren & $\begin{array}{l}\text { Vorinostat (SAHA), Trichostatin A, LBH-589, } \\
\text { Oxamflatin, Pyroxamid, PXD 101, LAQ824 } \\
\text { Scriptaid, Tubacin, SK-7041, SK-7068 }\end{array}$ \\
\hline $\begin{array}{l}\text { Kurzkettige } \\
\text { Fettsäuren }\end{array}$ & $\begin{array}{l}\text { Natroinsäure, Natriumbutyrat, } \\
\text { Zyklische Peptide }\end{array}$ \\
\hline Ketone & Depsipeptid (FK228), Trapoxin, Apicidin \\
\hline Benzamide & Trifluormethyl-Ketone, $\alpha$-Ketoamide \\
\hline
\end{tabular}

Drittlinientherapie mit Mykosis fungoides-Patienten des Stadiums IB-IVB nach mindestens zwei vorangegangen systemischen Therapien 2007 von der FDA zugelassen. In dieser Studie zeigten 30 Prozent der mehrfach vortherapierten Patienten eine Remission, weiterhin konnte eine Besserung des krankheitsassoziierten Pruritus beobachtet werden. [22]. Mittlerweile wurde die EU-Zulassung für Vorinostat beantragt.

In den bisher durchgeführten Studien fand sich keine Assoziation einer Vorinostat-Therapie mit opportunistischen Infektionen. $\mathrm{Zu}$ den bisher am häufigsten beschriebenen Nebenwirkungen zählen Müdigkeit, Thrombozytopenie und gastrointestinale Beschwerden. Die Applikation erfolgt oral, weiterhin wird von einem schnellen Wirkungseintritt berichtet [22,23]. Derzeit wird die Wirksamkeit von Vorinostat bei soliden Tumoren und unterschiedlichen hämatologischen Neoplasien evaluiert. Da bisher synergistische Effekte von Vorinostat in Kombination mit weiteren antineoplastischen Präparaten bei anderen Tumorentitäten beschrieben wurden [24-26], könnte die Kombination von Vorinostat mit etablierten Therapiemodalitäten kutaner T-Zell-Lymphome zu einem noch stärkeren Ansprechen führen. Depsipeptid wurde in einer Phase-I-Studie ebenfalls bei Patienten mit kutanen T-Zell-Lymphomen eingesetzt. Auch hier war ein Ansprechen zu beobachten, allerdings war die Therapie mit kardialen Nebenwirkungen assoziiert [27]. Derzeit wird die Wirksamkeit des Histondeacetylaseinhibitors APO 866 in einer
Phase-II-Studie bei Patienten mit rezidivierenden bzw. therapieresistenten kutanen T-Zell-Lymphomen evaluiert.

Ein weiterer Ansatzpunkt der zielgerichteten Therapie bei kutanen Lymphomen ist der Einsatz von spezifischen Antikörpern. Für kutane T-Zell-Lymphome sind hier die Zielstrukturen CD4 und CD52 auf T-Zellen zu nennen.

\section{Zanolimumab (HuMax-CD4)}

$\nabla$

Zanolimumab ist ein humaner Antikörper, der gegen den CD4-Rezeptor gerichtet ist. Hierdurch entfaltet er eine spezifische Wirkung gegen T-Helfer-Zellen und maligne Zellen kutaner T-Zell-Lymphome. Da eine Herunterregulierung oder ein Verlust der CD4-Expression maligner T-Zellen im Krankheitsverlauf kutaner T-Zell-Lymphome in der Regel nicht beobachtet wird, stellt dieses Oberflächenmolekül eine Zielstruktur in allen Krankheitsstadien dar. Eine Phase-I-Studie zeigte bereits 1991 eine Wirksamkeit des Antikörpers bei Patienten mit Mykosis fungoides [28]. Die Wirksamkeit von Zanolimumab konnte im Weiteren in zwei klinischen multizentrischen Phase-II-Studien bei Patienten in frühen und späten Krankheitsstadien bestätigt werden. Auch in Bezug auf Nebenwirkungen wird dieser Antikörper in den bisherigen Untersuchungen positiv bewertet. So wird vor allem über das Auftreten von nicht schwerwiegenden Infektionen und Ekzemen berichtet [29]. Aktuell wird Zanolimumab in einer klinischen Phase-III-Studie bei Mykosis fungoides-Patienten in fortgeschrittenen Krankheitsstadien evaluiert.

\section{Alemtuzumab (MabCampath ${ }^{\circledR}$ ) \\ $\nabla$}

Alemtuzumab ist ein humanisierter, gegen CD52 gerichteter, monoklonaler Antikörper. CD52 wird auf T- und B-Lymphozyten, Macrophagen, NK-Zellen und Monozyten exprimiert, jedoch nicht auf hämatopoetischen Stammzellen. Nach Bindung von Alemtuzumab an CD52 wird sowohl eine zellvermittelte als auch eine komplementvermittelte zytotoxische Reaktion ausgelöst. Der Einsatz von Alemtuzumab ist mittlerweile ein etabliertes Behandlungsverfahren der chronisch lymphatischen Leukämie vom B-Zell-Typ (B-CLL). Daneben wird die antientzündliche Wirkung des Antikörpers auch bei multipler Sklerose angewendet. Da maligne T-Zellen eine besonders starke Expression von CD52 aufweisen, wird der Antikörper bei unterschiedlichen T-Zell-Neoplasien eingesetzt. So zeigten sich unter anderem hohe Ansprechraten bei Patienten mit Prolymphozytenleukämie vom T-Zell-Typ (T-PLL) [30]. In einer klinischen Phase-II-Studie wurde die Wirksamkeit bei Patienten mit Mycosis fungoides der Stadien IIA bis IVB und Sézary-Syndrom evaluiert. Hier zeigte sich ein Gesamtansprechen von 55\%, davon 32\% komplette Remissionen [31]. Die unter Alemtuzumab beschriebenen Nebenwirkungen beinhalten unter anderem zytokin-induzierte Symptome wie Fieber, Urtikaria und Hypotonie, die durch eine Prämedikation, z.B. mit Antihistaminika, abgemildert werden können. Weiterhin kann Alemtuzumab zu einer, zum Teil Monate andauernden, T-Zell-Depletion und Myelosuppression führen. Dies bringt ein erhöhtes Risiko für opportunistische Infekte wie CMV-Reaktivierungen mit sich. Aus diesem Grund sind eine engmaschige Überwachung der betreffenden Patienten sowie die prophylaktische Gabe von Antibiotika und Virusstatika erforderlich. 


\begin{tabular}{|c|c|c|c|}
\hline & Studie 1 & Studie 2 & EBMT \\
\hline Behandlung & $\begin{array}{l}\text { Lenalidomid } \\
\text { Erhaltungstherapie }\end{array}$ & $\begin{array}{l}\text { Histondeacetylase-Inhibitor (HDI) } \\
\text { vs HDI + Proteinase-Inhibitor }\end{array}$ & $\begin{array}{l}\text { Allogene Stammzell-Trans- } \\
\text { plantation nach Konditionie- } \\
\text { rung mit reduzierter Intensität }\end{array}$ \\
\hline $\begin{array}{l}\text { Einschluss- } \\
\text { bedingung }\end{array}$ & $\begin{array}{l}\text { Stadium IIB-IV } \\
\text { Keine systemische } \\
\text { Chemotherapie } \\
\text { Ungeeignet für lokale } \\
\text { Behandlungen der Haut } \\
\text { PR/CR nach Nucleosid- } \\
\text { analogon } \pm \text { RT }\end{array}$ & $\begin{array}{l}\text { Stadium IIB-IV } \\
\text { Rezidiv/Progression nach } \\
\text { systemischer Chemotherapie } \\
\text { Ungeeignet für lokale } \\
\text { Behandlungen der Haut } \\
\text { Keine Vortherapie mit HDI oder } \\
\text { Proteinase-Inhibitor }\end{array}$ & $\begin{array}{l}\text { Stadium IIB-IV } \\
\text { Rezidiv/Progression nach } \\
\text { systemischer Chemotherapie }\end{array}$ \\
\hline
\end{tabular}

Tab. 2 Therapieplattform der EORTC-CLTF (cutaneous lymphoma task force) bei fortgeschrittenen kutanen T-ZellLymphomen.

\section{Therapieplattform der EORTC-CLTF \\ $\nabla$}

Für die Behandlung der kutanen T-Zell-Lymphome ist ein stadiengerechtes Vorgehen zu empfehlen [32,33]. Haut-gerichtete Therapien wie PUVA oder UVB Schmalband $311 \mathrm{~nm}$ haben hohe Ansprechraten (60-80\%) und selbst Rezidive können mit dem gleichen Therapieschema oder ggf. in Kombination mit Interferon-alpha $2 \mathrm{a}$ wieder erfolgreich behandelt werden. Kommt es zu therapierefraktären Plaques oder Tumoren können eine Röntgenweichstrahltherapie oder eine Bestrahlungstherapie mit schnellen Elektronen erfolgreich angewandt werden. Die Behandlung im Tumorstadium, bei Erythrodermie oder mit Lymphknotenbeteiligung/Organbeteiligung gehört in eine erfahrene dermatoonkologische Betreuung, da in diesen Fällen eine systemische Therapie mit Bexaroten, Doxirubicin, Denileukin Diftitox, Vorinostat oder die extrakorporale Photopherese zum Einsatz kommt [34]. Die Behandlung bei mehrfachen Rezidiven oder therapierefraktären Fällen erfordert Therapieschemata, die individuell auf den Patienten abgestimmt sind und neben dem palliativen Behandlungserfolg auch die Nebenwirkungsprofile und die Lebensqualität berücksichtigen.

Im Rahmen des EORTC Cutaneous Lymphoma Task Force Clinical Meeting in Kopenhagen 2008 wurde eine Therapieplattform für fortgeschrittene kutane T-Zell-Lymphome eingeführt ( $\bullet$ Tab. 2). Diese Initiative ist in vielfacher Hinsicht von Bedeutung. Die Therapiemöglichkeiten bei fortgeschrittenen kutanen T-ZellLymphomen basieren meist of empirischen Daten von Studien mit geringer Fallzahl, sodass bei multizentrischen Studien der EORTC erheblich höhere Stichproben erhoben werden können. Die Studienprotokolle sind aufeinander abgestimmt, damit es bei sukzessiver Anwendung nicht zu typischen kumulativen Toxizitäten gleicher Substanzgruppen kommen kann und schließlich entsteht ein Studiennetzwerk als Grundlage für die klinische und translationale wissenschaftliche Forschung. Auf Wunsch des EORTC CLTF taske force-Komitees dürfen im Folgenden noch nicht alle Studiensubstanzen genannt werden, bevor die Freigabe der Studien endgültig erfolgt ist.

Die erste Therapieoption bei fortgeschrittenen T-Zell-Lymphomen (Stadium IIB - IV) ist vorgesehen für Patienten ohne bisher durchgeführte Polychemotherapie (Protokoll 21081). Studienmedikation ist Lenalidomid ein Substanzanalog des Thalidomid, mit anti-angiogener, anti-entzündlicher und kostimmulatorischer immunologischer Aktivität. Lenalidomid soll hier als Erhaltungstherapie am Ende ein Initiierungstherapie („debulking“) mit einem Nukleosidanalogon \pm Radiotherapie durchgeführt werden. Dabei sind 4 Therapiezyklen in 4-wöchigen Abständen für vorgesehen. Kommt es dabei zu einem Ansprechen (CR/PR), wird der Patient randomisiert in einem Beobachtungs- arm oder einen Therapieerhaltungsarm mit Lenalidomid ( $25 \mathrm{mg}$ oral/Tag für 3 Wochen/Monat). Kommt es zu einem weiteren Rezidiv oder wurde vorher bereits eine Polychemotherapie durchgeführt, bietet sich das Protokoll 21082 an. Bei dieser Studie wird untersucht, ob ein Histondeacetylase-Inhibitor in Kombination mit dem Proteinaseinhibitor effektiver als die Monotherapie ist. Die Rationale ist hier, dass der Proteinaseinhibitor synergistische Effekte entwickelt und das rezidivfreie Intervall verlängert. Präklinische Daten konnten bereits bei der Kombinationstherapie die Induktion einer erhöhten Apoptoserate bei Lymphomzellen zeigen. In einem weiteren Schritt können Patienten mit fortgeschrittenen kutanen T-Zell-Lymphomen einem Behandlungsprotokoll der European Group for Blood and Marrow Transplant (EBMT) zugeführt werden. Es handelt sich dabei um eine allogene hämatopoetische Stammzelltransplantation mit einer Konditionierung mit reduzierter Intensität. Bedingung ist hier, dass bereits eine Chemotherapie durchgeführt wurde und es in diesem Verlauf zu einem Rezidiv oder einer Progression der Erkrankung gekommen ist.

Zusammenfassend gibt es einige vielversprechende zielgerichtete Therapieoptionen für die Behandlung der kutanen T-Zell-Lymphome. Angesichts der neuen Möglichkeiten begrüßen wir die Initiative der EORTC-CLTF zur Koordination weiterer Studien zur Gewinnung einer verlässlichen Datengrundlage für leitliniengerechte Therapien.

\section{Abstract}

\section{New Treatment Strategies for Cutaneous T-Cell Lymphomas \\ $\nabla$}

Cutaneous T-cell lymphomas are a group of extranodal nonHodgkin lymphomas which show a broad heterogenity regarding clinical and histological manifestations in the skin. Current research has confirmed the heterogentity of cutaneous T-cell lymphomas and has given insight into their biology, immunology and molecular genetics. The knowledge of target structures of malignant T-cells has opend new pathways in developing new therapeutic strategies. The accurate classification of specific clinical entities and specific information regarding individual patients is necessary in order to choose a targeted therapy. However, since a curative treatment is currently not available, the commonly used stage-adapted therapy still remains the recommended approach. 


\section{Literatur}

1 Willemze R, Jaffe ES, Burg G et al. WHO-EORTC classification for cutaneous lymphomas. Blood 2005; 105: 3768 - 3785

2 Olsen E, Vonderheid E, Pimpinelli $N$ et al. Revisions to the staging and classification of mycosis fungoides and Sezary syndrome: A proposal of the International Society for Cutaneous Lymphomas (ISCL) and the cutaneous lymphoma task force of the European Organization of Research and Treatment of Cancer (EORTC). Blood 2007; 110: 17131722

3 Kim YH, Willemze R, Pimpinelli $N$ et al. TNM classification system for primary cutaneous lymphomas other than mycosis fungoides and Sezary syndrome: a proposal of the International Society for Cutaneous Lymphomas (ISCL) and the Cutaneous Lymphoma Task Force of the European Organization of Research and Treatment of Cancer (EORTC). Blood 2007; 110: 479-484

4 Klemke CD, Goerdt S, Schrama D, Becker JC. New insights into the molecular biology and targeted therapy of cutaneous T-cell lymphomas. JDDG 2006; 5: 395 - 406

5 Kondapalli L, Soltani $K$, Lacouture ME. The promise of molecular targeted therapies: protein kinase inhibitors in the treatment of cutaneous malignancies. J Am Acad Dermatol 2005; 53: 291 - 302

6 van Doorn R, Zoutman WH, Dijkman R et al. Epigenetic Profiling of Cutaneous T-Cell Lymphoma: Promoter Hypermethylation of Multiple Tumor Suppressor Genes Including BCL7a, PTPRG, and p73. J Clin Oncol 2005; 23: 3886-3896

7 Booken N, Gratchev A, Utikal J et al. Sézary syndrome is a unique cutaneous T-cell lymphoma as identified by an expanded gene signature including diagnostic marker molecules CDO1 and DNM3. Leukemia 2008; 22: 393-399

8 Duvic M, Martin AG, Kim Yet al. Phase 2 and 3 clinical trial of oral bexarotene (Targretin capsules) for the treatment of refractory or persistent early-stage cutaneous T-cell lymphoma. Arch Dermatol 2001; 137: $581-593$

9 Duvic M, Hymes $K$, Heald $P$ et al. Bexarotene is effective and safe for treatment of refractory advanced-stage cutaneous T-cell lymphoma: multinational phase II-III trial results. J Clin Oncol 2001; 19: 2456 2471

10 Olsen E, Duvic M, Frankel A et al. Pivotal phase III trial of two dose levels of denileukin diftitox for the treatment of cutaneous T-cell lymphoma. J Clin Oncol 2001; 19: 376 - 388

11 Foss FM, Waldmann TA. Interleukin-2 receptor-directed therapies for cutaneous lymphomas. Hematol Oncol Clin North Am 2003; 17: $1449-1458$

12 Foss F, Demierre MF, DiVenuti G. A phase- 1 trial of bexarotene and denileukin diftitox in patients with relapsed or refractory cutaneous T-cell lymphoma. Blood 2005; 106: 454-457

13 Hagberg H, Pettersson M, Bjerner T, Enblad G. Treatment of a patient with a nodal peripheral T-cell lymphoma (angioimmunoblastic T-Cell lymphoma) with a human monoclonal antibody against the CD4 antigen (Hu-Max-CD4). Med Oncol 2005; 22: 191 - 194

14 Piekarz RL, Robey R, Sandor $V$ et al. Inhibitor of histone deacetylation, depsipeptide (FR901228), in the treatment of peripheral and cutaneous T-cell lymphoma: A case report. Blood 2001; 98: 2865-2868

15 Sandor V, Bakke S, Robey RW et al. Phase I trial of the histone deacetylase inhibitor, depsipeptide (FR901228, NSC 630176), in patients with refractory neoplasms. Clin Cancer Res 2002; 8: 718-728
16 Groll M, Berkers CR, Ploegh HD, Ovaa H. Crystal structure of the boronic acid-based proteosome inhibitor Bortezomib in complex with yeast 20S proteosome. Structure 2006; 14: 451 - 456

17 O'Connor OA. Targeting histones and proteosomes: New strategies for treatment of lymphoma. J Clin Oncol 2005; 23: 6429-6436

18 Zinzani PL, Musuraca G, Tani M et al. Phase II trial of proteasome inhibitor bortezomib in patients with relapsed or refractory cutaneous T-cell lymphoma. J Clin Oncol 2007; 25: 4293-4297

19 Lindemann RK, Gabrielli B, Johnstone RW. Histonedeacetylase inhibitors for the treatment of cancer. Cell Cycle 2004; 3: 779-788

20 Johnstone $R W$. Histone-deacetylase inhibitors: novel drugs for the treatment of cancer. Nat Rev Drug Discov 2002; 1: 287-299

21 Zhang C, Richon V, Ni X et al. Selective induction of apoptosis by histone deacetylase inhibitor SAHA in cutaneous T-cell lymphoma cells: Relevance to mechanism therapeutic action. J Invest Dermatol 2005; 125: $1045-1052$

22 Olsen E, Kim YH, Kuzel TM et al. Phase IIB multicenter trial of vorinostat in patients with persistent, progressive or treatment-refractory cutaneous T-cell lymphoma. J Clin Oncol 2007; 25: 3109-3115

23 Duvic M, Talpur R, Ni X et al. Phase 2 trial of oral Vorinostat (suberoylanilide hydroxamic acid, SAHA) for refractory cutaneous T-cell lymphoma (CTCL). Blood 2007; 109: 31 - 39

24 Almenara J, Rosato R, Grant S. Synergistic induction of mitochondrial damage and apoptosis in human leukemia cells by flavopiridol and the histone deacetylase inhibitor suberoylanilide hydroxamic acid (SAHA). Leukemia 2002; 16: 1331 - 1343

25 Machion DC, Bicaku E, Daud AI et al. Sequence-specific potentiation of topoisomerase II inhibitors by the histone deacetylase inhibitor suberoylanilide hydroxamic acid. J Cell Biochem 2004; 92: 223 - 237

26 Lakshmikanthan V, Kaddour-Djebbar I, Lewis RW, Kumar MV. SAHAsensitized prostate cancer cells to TNF-alpha-related apoptosis-inducing ligand (TRAIL): Mechanisms leading to synergistic apoptosis. Int J Cancer 2006; 119: 221 - 228

27 Piekarz RL, Robey R, Sandor V. Inhibitor of histone deacetylation, depsipeptide (FR901228), in the treatment of peripheral and cutaneous T-cell lymphoma: A case report. Blood 2001; 98 (9): 2865 - 2868

28 Knox SJ, Levy R, Hodgkinson S et al. Observations on the effect of chimeric anti-CD4 monoclonal antibody in patients with mycosis fungoides. Blood 1991; 77: 20 - 30

$29 \mathrm{Kim}$ YH, Duvic M, Obitz E et al. Clinical efficacy of Zanolimumab (HuMax-CD4): two phase 2 studies in refractory cutaneous T-cell lymphoma. Blood 2007; 109: 4655-4662

30 Dearden CE, Matutes E, Cazin B et al. High remission rate in T-cell prolymphocytic leukemia with CAMPATH-1H. Blood 2001; 98: 1721 1726

31 Lundin J, Hagberg H, Repp R et al. Phase 2 study of alemtuzumab (antiCD52 monoclonal antibody) in patients with advanced mycosis fungoides/Sezary syndrome. Blood 2003; 101: 4267-4272

32 Dippel E, Gellrich S, Klemke CD et al. Kutane T-Zell Lymphome. JDDG 2003; 1 : $965-981$

33 Assaf C, Hummel M, Steinhoff $M$ et al. Early TCR-beta and TCR-gamma PCR detection of T-cell clonality indicates minimal tumor disease in lymph nodes of cutaneous T-cell lymphoma: Diagnostic and prognostic implications. Blood 2005; 105: $503-510$

34 Dippel E, Klemke CD, Goerdt S. Current status of cutaneous T-cell lymphoma: Molecular diagnosis, pathogenesis, therapy and future directions. Onkologie 2003; 26: 477-483 